\title{
Comparative utility of anti-citrullinated protein antibodies verses rheumatoid factor in the management of rheumatoid arthritis
}

Volume 8 Issue 4 - 2017

Anjali Patwardhan
Department of Child Health, University of Missouri, USA

Correspondence: Anjali Patwardhan MD, MRCPCH, MRCPI, MSc, MBA, IA, Assistant professor \& Director of Pediatric Rheumatology, Department of Child Health, University of Missouri, School of Medicine, 400 Keene Street, Suite $102 \mathrm{H}$ Columbia, Missouri 65212, USA, Tel 16142662187, Email patwardhana@health.missouri.edu

Received: April 27, 2017 | Published: July 13, 2017

fibrinogen, Deiminated Epstein-Barr Virus Nuclear Antigen 1 and vimentin..$^{2-4}$ Over the years, the assays using genetically modified citrullinated Vimentin antigen protein were developed to make them more standardized and efficient. These new tests include mutated citrullinated Vimentin (MCV-assay), filaggrin-derived peptides (CCPassay) and viral citrullinated peptides (VCP-assay). ${ }^{5}$ Interestingly it has same diagnostic performance as is of RF and anti-CCP, it is especially useful in the diagnosis of RF \& Anti -CCP negative RA patients. It has not been studied well in the pediatric population as yet.

The 2010 ACR-EULAR classification criteria for rheumatoid arthritis included ACPA in the diagnostic criteria, and ACPA testing played a significant part in the classification system of RA also. ${ }^{6,7}$ Anti-Cyclic Citrullinated Peptide (ACCP) antibodies are one of the antibodies in the ACPA group, but interestingly all antibodies in this group not necessarily cross react with each other. ${ }^{8}$

In the pediatric population where the juvenile onset idiopathic rheumatoid arthritis is the most common rheumatic diseases of childhood, there is an absence of specific biomarkers for the diagnosis. A tiny pediatric population with JIA is seropositive (RF positive and Anti-Citrullinated Protein Antibodies (ACPAS) positive). This situation takes away the opportunity of early and aggressive therapy which is known to impact long-term morbidity and outcomes. It is also the fact that biomarkers are not well researched and explored in the pediatric population as they are in adult patients. Interestingly as it is evident in the classification of JIA, it is automatically presumed that only polyarthritis patients can be rheumatoid factor positive (RF-positive and RF-negative polyarthritis). There are only a few biomarkers that are tested using standardized universally accepted tests which, so that results from different geographical areas can be compared. These biomarkers include Rheumatoid Factor (RF) /RF isotypes and Anticyclic citrullinated peptide antibodies/ anti-CCP isotopic antibodies (Anti-CCP).

Citrullinated proteins and peptides are never present in normal joints or blood unless there is autoimmune inflammation ongoing on. ${ }^{9}$

There were several antigenic proteins later recognized which were similar to Vimentin which also reacted with autoantibodies identified in RA patients. The examples of few such proteins are 
The antiperinuclear factor (APF) and anti-keratin antibodies (AKA) were the first two members of ACPA family, and they were seen to recognize citrullinated epitopes of filaggrin peptide. Later on, based on these facts the first (Anti-CCP), the second (Anti-CCP2) and now third generation anti-cyclic citrullinated peptide (anti-CCP3) antibody assays were developed.

To detect the citrullinated antibodies, various manufacturers use different citrullinated antigen substrates such as Epstein-Barr virusor IgG-derived peptides, synthetic cyclic peptides, mutated human vimentin, and, recombinant rat filaggrin. These manufacturers though have little difference in their characteristics of analytical methods such as titrating various sensitivities for a fixed specificity, they all perform testing by enzymatic immunoassay (EIA) methods. ${ }^{10}$ Research has shown that the diagnostic accuracy and analytical imprecision can be influenced significantly by the antigenic source used in the test. ${ }^{10}$ The sensitivity and specificity of different manufacturers may vary which is based on their selected values of cut off. For example, at a fixed specificity of ' $\mathrm{X}$,' the sensitivities ranged from ' $\mathrm{Y}$ ' to ' $\mathrm{Z}$ ' for different manufacturer's tests. False-positive results are common in patients who had or were recovering from viral syndromes. ${ }^{10}$

The vimentin is a relatively new but considered a very reliable and standard autoantigen. Vimentin is a naturally occurring antigen as oppose to several others artificially developed for the test. Vimentin is secreted from dying macrophages and then citrullinated also by macrophages during the process of apoptosis, or by proinflammatory cytokines (tumor necrosis factor-alpha: TNF-alpha) during the inflammation process. ${ }^{11}$ To improve further the accuracy and specificity a genetically modified citrullinated vimentin (MCV) is used as an antigen to identify auto antibodies. ${ }^{12,13}$

CCP antibody testing may also be ordered to help evaluate the likely development of RA in people with undifferentiated arthritis or test the possibility of RA in the relatives of RA patients the familial incidence is not very significant. In Monozygotic Twins the incidence is $15.4 \%$ while in dizygotic twins it is $3.8 \%$. According to ACR, approximately $95 \%$ of those with a positive CCP antibody will die eventually in future will develop after a variable and uncertain period of latency. It goes without saying that early detection of RA is essential for guiding appropriate treatment decisions and can affect outcomes. Therefore, Anti-Citrullinated Protein Antibodies (ACPAS) is used for undifferentiated arthritis and early detection when clinical suspicion is high where RF is not useful. ${ }^{14}$ The RF is used as a screening test, but Anti-CCP cannot be used. As mentioned before Anti -CCP can predate the RA by several years while RF does not predate the disease.

Both RF Anti-Citrullinated Protein Antibodies (ACPAS) have high positive predictive value in predicting erosive disease if they are present at onset. Anti-Citrullinated Protein Antibodies (ACPAS) has a higher positive predictive value for erosive disease as compared to $\mathrm{RF}^{5,14,15}$

The sensitivity of RF is marginally better than Anti-Citrullinated Protein Antibodies (ACPAS) in the diagnosis of the RA (69\% VS $67 \%$ in most of the meta-analysis) while the Specificity of AntiCitrullinated Protein Antibodies (ACPAS) in the diagnosis of RA is better than that of RF.

Several immunogenic viruses are known to produce false positive reactions or even positive tests due to the production of polyclonal -B-Cell reaction such as hepatitis- $\mathrm{C}$ virus (HCV)associated cryoglobulinemia. The possibility of RF being positive in these situations is higher than Anti-Citrullinated Protein Antibodies (ACPAS) being positive.
The titer of both the antibodies decreases with treatment ${ }^{16}$ but never disappear (PMID). The titers of RF antibodies and associated diseases are more responsive to infliximab or any other TNF- inhibitor therapy as compared to Anti-Citrullinated Protein Antibodies (ACPAS) titers and associated disease. ${ }^{17-21}$ The positive RF can be used as an early predictor of the efficacy of anti-TNF therapy. ${ }^{22}$

The HLA-DRB1 alleles carrying the shared epitope (SE) association with Anti-Citrullinated Protein Antibodies (ACPAS) autoantibodies but not with RF antibodies. ${ }^{23-27}$

The RF antibodies have the correlation with Th1/Th2 cytokines while Anti-Citrullinated Protein Antibodies (ACPAS) antibodies do not have any such relationship. ${ }^{23}$

As it is known, RF antibodies can be present in several other systemic autoimmune diseases such as in lupus, Graves' disease and Sjogren's syndrome, etc. while Anti-Citrullinated Protein Antibodies (ACPAS) is not necessarily positive in these diseases. ${ }^{28}$ On the other hand, Anti-Citrullinated Protein Antibodies (ACPAS) can be positive in active systemic or pulmonary tuberculosis Positive in Tuberculosis.

Apparently, RF can be recommended as a screening test for the diagnosis of RA while ideally, Anti-Citrullinated Protein Antibodies (ACPAS) is not.

Summarizing the communication on the comparative utility of RF verses in the management of RA, clearly Anti-Citrullinated Protein Antibodies (ACPAS) show better value in making the diagnosis of RA and predicting outcomes and even selecting the therapy options based on the responsiveness. It is amazing fact that RF and Anti-Citrullinated Protein Antibodies (ACPAS) apparently are not the similar spectrum of antibodies. Both of these antibodies may be representatives of different RA diseases with specific diseases course prognosis and drug responsiveness. Therefore, RF and Anti-Citrullinated Protein Antibodies (ACPAS) cannot be used synonymously or could be used interchangeable as they represent different diseases and outcomes.

\section{Conflicts of Interests}

There is no conflict of interest in composing this manuscript.

\section{Acknowledgments}

None.

\section{References}

1. Waaler E. On the occurrence of a factor in human serum activating the specific agglutintion of sheep blood corpuscles. 1939. APMIS 2007;115(5):422-439.

2. Vossenaar ER, Normand D, Elvy L, et al. Rheumatoid arthritis specific anti-Sa antibodies target citrullinated vimentin. Arthritis Res Ther. $2004 ; 6(2): 142-150$

3. Pratesi F, Tommasi C, Anzilotti C, et al. Deiminated Epstein-Barr virus nuclear antigen 1 is a target of anti-citrullinated protein antibodies in rheumatoid arthritis. Arthritis Rheum. 2006;54(3):733-741.

4. Bang H, Egerer K, Gauliard A, et al. Mutation and citrullination modifies vimentin to a novel autoantigen for rheumatoid arthritis. Arthritis Rheum. 2007;56(8):2503-2511.

5. Nishimura K, Sugiyama D, Kogata Y, et al. Meta-analysis: diagnostic accuracy of anti-cyclic citrullinated peptide antibody and rheumatoid factor for rheumatoid arthritis. Ann Intern Med. 2007;146(11):797-808.

6. Mueller RB, M SchiffT, Kaegi A, et al. The new 2010 ACR/EULAR criteria as predictor of clinical and radiographic response in patients with early arthritis. Clin Rheumatol. 2015;34(1):51-59. 
7. van der Heijde D, Helm-van Mil AH, Aletaha D, et al. EULAR definition of erosive disease in light of the 2010 ACR/EULAR rheumatoid arthritis classification criteria. Ann Rheum Dis. 2013;72(4):479-481.

8. Ioan-Facsinay A, el-Bannoudi H, Scherer HU, et al. Anti-cyclic citrullinated peptide antibodies are a collection of anti-citrullinated protein antibodies and contain overlapping and non-overlapping reactivities. Ann Rheum Dis. 2011;70(1):188-193.

9. Klareskog L, Widhe M, Hermansson M, et al. Antibodies to citrullinated proteins in arthritis: pathology and promise. Curr Opin Rheumatol. 2008;20(3):300-305.

10. Bizzaro N, Tonutti E, Tozzoli R, et al. Analytical and diagnostic characteristics of 11 2nd- and 3rd-generation immunoenzymatic methods for the detection of antibodies to citrullinated proteins. Clin Chem. 2007;53(8):1527-1533.

11. van der Helm-van Mil AH, Verpoort KN, Breedveld FC, et al. Antibodies to citrullinated proteins and differences in clinical progression of rheumatoid arthritis. Arthritis Res Ther. 2005;7(5):R949-R958.

12. Budhram A, Chu R, Rusta-Sallehy S, et al. Anti-cyclic citrullinated peptide antibody as a marker of erosive arthritis in patients with systemic lupus erythematosus: a systematic review and meta-analysis. Lupus. 2014;23(11):1156-1163.

13. Shovman O, Gilburd B, Zandman-Goddard G, et al. The diagnostic utility of anti-cyclic citrullinated peptide antibodies, matrix metalloproteinase-3, rheumatoid factor, erythrocyte sedimentation rate, and $\mathrm{C}$-reactive protein in patients with erosive and non-erosive rheumatoid arthritis. Clin Dev Immunol. 2005;12(3):197-202.

14. Schattner A. Review: anti-cyclic citrullinated peptide antibody is a more specific test for rheumatoid arthritis than rheumatoid factor. ACP J Club. 2007;147(3):78

15. Roubenoff R, Beckman E, Weinblatt M, et al. Biological significance of anti-cyclic citrullinated peptide antibody in rheumatoid arthritis. Ann Intern Med. 2008;148(5):403-404.

16. Vis M, Bos WH, Wolbink G, et al. IgM-rheumatoid factor, anticyclic citrullinated peptide, and anti-citrullinated human fibrinogen antibodies decrease during treatment with the tumor necrosis factor blocker infliximab in patients with rheumatoid arthritis. $J$ Rheumatol. 2008;35(3):425-428.

17. De Rycke L, Verhelst X, Kruithof E, et al. Rheumatoid factor, but not anti-cyclic citrullinated peptide antibodies, is modulated by infliximab treatment in rheumatoid arthritis. Ann Rheum Dis. 2005;64(2):299-302.
18. Russell AS, WP Maksymowych, M de Silva. Anti-cyclic citrullinated peptide antibodies in patients with rheumatoid arthritis treated with antitumour necrosis factor agents. Ann Rheum Dis. 2005;64(12):1807.

19. Caramaschi P1, Biasi D, Tonolli E, et al. Antibodies against cyclic citrullinated peptides in patients affected by rheumatoid arthritis before and after infliximab treatment. Rheumatol Int. 2005;26(1):58-62.

20. Bobbio-Pallavicini F, Caporali R, Bugatti S, et al. What can we learn from treatment-induced changes in rheumatoid factor and anticitrullinated Peptide antibodies? J Rheumatol. 2008;35(10):1903-1935.

21. Bruns A, Nicaise-Roland P, Hayem G, et al. Prospective cohort study of effects of infliximab on rheumatoid factor, anti-cyclic citrullinated peptide antibodies and antinuclear antibodies in patients with longstanding rheumatoid arthritis. Joint Bone Spine. 2009;76(3):248-253.

22. Hayashi N, Kumagai S. Anti-cyclic citrullinated peptide antibodies and rheumatoid arthritis. Rinsho Byori. 2010;58(5):466-479.

23. Correa PA, Tobón GJ, Citera G, et al. Anti-cyclic citrullinated peptide antibodies in rheumatoid arthritis: relation with clinical features, cytokines and HLA-DRB1. Biomedica. 2004;24(2):140-152.

24. Huizinga TW, Amos CI, van der Helm-van Mil AH, et al. Refining the complex rheumatoid arthritis phenotype based on specificity of the HLA-DRB1 shared epitope for antibodies to citrullinated proteins. Arthritis Rheum. 2005;52(11):3433-3438.

25. van der Helm-van Mil AH1, Verpoort KN, le Cessie S, et al. The HLADRB1 shared epitope alleles differ in the interaction with smoking and predisposition to antibodies to cyclic citrullinated peptide. Arthritis Rheum. 2007;56(2):425-432.

26. Kapitány A, Szabó Z, Lakos G, et al. Associations between serum antiCCP antibody, rheumatoid factor levels and HLA-DR4 expression in Hungarian patients with rheumatoid arthritis. Isr Med Assoc J. 2008;10(1):32-36

27. de Vries RR, TW Huizinga, RE Toes. Redefining the HLA and RA association: to be or not to be anti-CCP positive. $J$ Autoimmun. 2005;25Suppl:21-25.

28. Niewold TB, MJ Harrison, SA Paget. Anti-CCP antibody testing as a diagnostic and prognostic tool in rheumatoid arthritis. QJM. 2007;100(4):193-201. 